\title{
Design of Automatic Following and Locating Electric Carrier Based on Ultrasonic Positioning and PI Controller
}

\author{
Junyang Tang ${ }^{1,}$, Jundi Wang ${ }^{2, b}$,Weipeng Lü ${ }^{2}$ and Dongdong Chen ${ }^{2}$ \\ ${ }^{1}$ Nanjing Foreign Language School, Nanjing 210000, China \\ ${ }^{2}$ Mengfu Education Technology Co.Ltd , Shanghai 200007, China \\ atjy_1212@126.com, bcourse@mfeducation.com
}

\begin{abstract}
Keywords: ultrasonic positioning, automatic following and locating, PI algorithm, intelligent universal wheels,
\end{abstract} the least squares optimization method.

\begin{abstract}
Ultrasonic devices have lots of uses in many fields, but they are rather limited in the indoor positioning fields. In order to solve the problems of the present ultrasonic positioning devices, this paper illustrates an Ultrasonic Positioning and Analysis System for Automatic following and Locating Electric Carrier called UPASAFLEC in short. This paper adopts the least squares optimization method together with PI control. Highly accurate measurement and precise speed control are the advantages. This paper will mainly go through the design of the software and hardware of the UPASAFLEC and prove it useful with some experiments.
\end{abstract}

\section{Introduction}

There are a variety of ways to locate and position a particular object. The tools that people usually use like the Global Positioning System, the BeiDou Navigation Satellite System or the GLONASS, are unable to help to deal with indoor positioning. However, few of them would use ultrasonic technology to do so.

Ultrasonic positioning systems are not at a very developed or advanced state. The structure of the Improved Active Bat Indoor Ultrasonic Positioning Method [5] is very simple and the RF free ultrasonic positioning [3] is relatively accurate. They are small and potable whereas they can be easily affected by the nearby noise or the errors. The Pseudo-orthogonal Chirp-based Multiple Ultrasonic Transducer positioning system [2] and the Moving Target Tracking Algorithm Based on APRBA Particle Filter Algorithm [8] are very accurate and good at avoiding the interference of extra ultrasonic waves. However, their algorithms are too complex and hard to be simplified. Though the calculating of mobile ultrasonic transducer positioning [4] is quite advanced and the construction of the hardware of the Navigation Device [10] is very simple, they both have a very complex theory and a way of analyzing received data. The DOLPHIN system [1] is able to position the objects with minimal manual configuration. Moreover, the system requires only a few preset reference nodes to locate all the nodes in the system. However, this system is easy to lead to node and recognition failures. Although the Blind Guiding System Based on Ultrasonic Positioning [7] is much convenient, it is too reliant on the GPS system. The independent following robot [9] is much different from the Blind Guiding System Based on Ultrasonic Positioning [7], whose construction is not a simple work; in contrast the errors are few. A better one might be the Intelligent Following Carriage [6] which uses a very simple algorithm. However, the structure of the wheels makes it rather inconvenient.

From this point of view, this paper has shown a better and a more convenient method which is called the UPASAFLEC (Ultrasonic Positioning and Analysis System for Automatic following and Locating Electric Carrier). The carrier uses an ultrasonic positioning system, the least squares optimization method and Arduino's hardware. Together with the PI algorithm and a positioning algorithm, this paper showed, the carrier is enable to receive the ultrasonic signal transmitted by an 
ultrasonic transmitter on its owner, then locates the position of its owner and follows the owner automatically.

This paper will mainly go through the structure and the construction of the hardware for UPASAFLEC in the next section. In the third section, the paper will summarize the algorithms and the theories of the UPASAFLEC and will discuss the experimentation and results in the fourth section. For the last section, this paper will make a conclusion of the UPASAFLEC and summarize the entire essay.

\section{The Structure and the Construction of the Hardware for UPASAFLES}

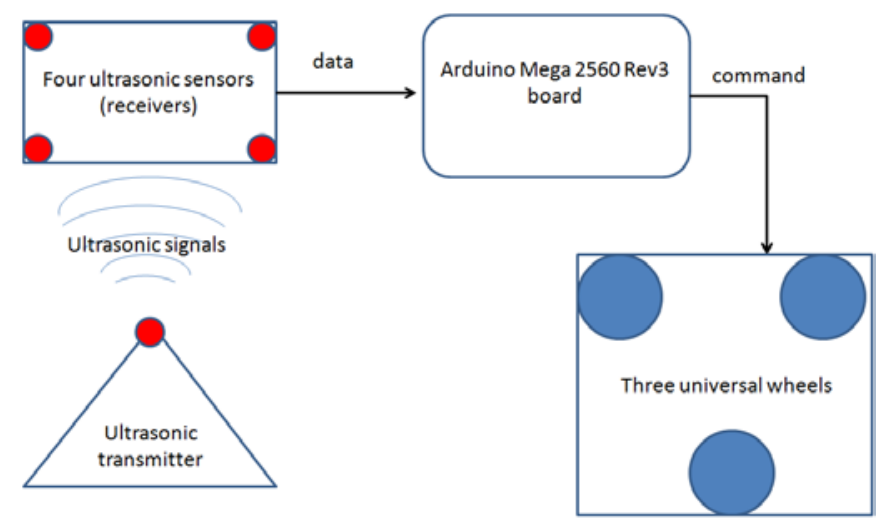

Fig.1 System structure

Figure 1 shows the structure of the UPASAFLEC hardware. The hardware system consists of an Arduino Mega 2560 Rev3 board, four ultrasonic sensors and three Universal wheels. The four ultrasonic sensors are on the top of the carrier to receive data sent by the transmitter. The received data then goes to the Arduino Mega 2560 Rev3 board which is in the centre, processing and analyzing the data. Afterwards the data is processed into the different velocity of the three universal wheels. Because of the decomposition and synthesis of velocities, the carrier will go to the direction as it should go.

The four receiving ultrasonic sensors receive the data (ultrasonic signal) from a transmitter which is attached to the carrier owner's back or waist. The transmitter mainly consists of an Arduino Nano, an ultrasonic sensor and batteries. The transmitter is used to give out ultrasonic signals in order to let the receiving sensors track the owner more conveniently and automatically.

There are two main advantages in this mechanism. Firstly, there are four receiving sensors which greatly improve the accuracy of the positioning process. Secondly, the wheels are universal wheels which make turning much more convenient.

\section{Algorithms and the Theories}

\subsection{Ultrasonic Positioning Algorithm}

At first, a three - dimensional coordinate system is built as figure 2: 


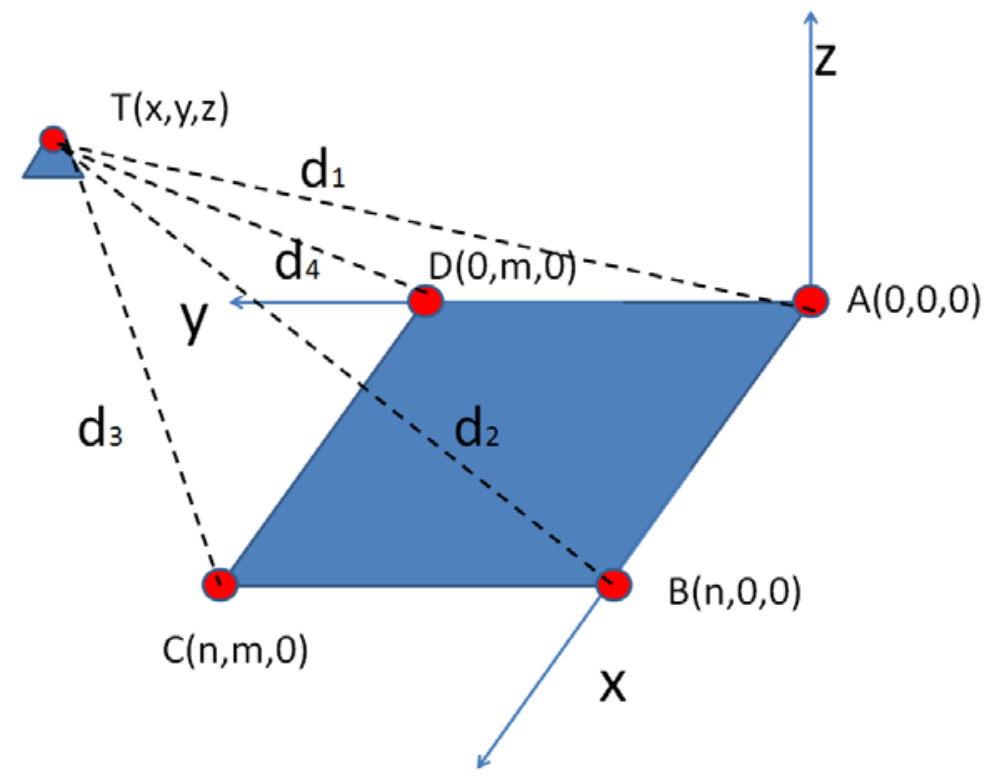

Fig.2 Three - dimensional coordinate system built in this paper As a result, define the following equations:

$\left(x_{1}, y_{1}, z_{1}\right)=(0,0,0),\left(x_{2}, y_{2}, z_{2}\right)=(n, 0,0),\left(x_{3}, y_{3}, z_{3}\right)=(0, m, 0),\left(x_{3}, y_{3}, z_{3}\right)=(n, m, 0)$

The distance from the transmitter to the receivers $\left(d_{1}, d_{2}, d_{3}, d_{4}\right)$ can be got by using the ultrasonic sensors.

As a result:

$\left\{\begin{array}{l}\left(x-x_{1}\right)^{2}+\left(y-y_{1}\right)^{2}+\left(z-z_{1}\right)^{2}=d_{1}^{2} \\ \left(x-x_{2}\right)^{2}+\left(y-y_{2}\right)^{2}+\left(z-z_{2}\right)^{2}=d_{2}^{2} \\ \left(x-x_{3}\right)^{2}+\left(y-y_{3}\right)^{2}+\left(z-z_{3}\right)^{2}=d_{3}^{2} \\ \left(x-x_{4}\right)^{2}+\left(y-y_{4}\right)^{2}+\left(z-z_{4}\right)^{2}=d_{4}^{2}\end{array}\right.$

Then do a subtract linearization:

$$
\left\{\begin{array}{l}
\left(x_{4}-x_{1}\right) x+\left(y_{4}-y_{1}\right) y+\left(z_{4}-z_{1}\right) z=\frac{1}{2}\left[d_{1}^{2}-d_{4}^{2}+x_{4}^{2}+y_{4}^{2}+z_{4}^{2}-\left(x_{1}^{2}+y_{1}^{2}+z_{1}^{2}\right)\right] \\
\left(x_{4}-x_{2}\right) x+\left(y_{4}-y_{2}\right) y+\left(z_{4}-z_{2}\right) z=\frac{1}{2}\left[d_{2}^{2}-d_{4}^{2}+x_{4}^{2}+y_{4}^{2}+z_{4}^{2}-\left(x_{2}^{2}+y_{2}^{2}+z_{1}^{2}\right)\right] \\
\left(x_{4}-x_{3}\right) x+\left(y_{4}-y_{3}\right) y+\left(z_{4}-z_{1}\right) z=\frac{1}{2}\left[d_{3}^{2}-d_{4}^{2}+x_{4}^{2}+y_{4}^{2}+z_{4}^{2}-\left(x_{3}^{2}+y_{3}^{2}+z_{3}^{2}\right)\right]
\end{array}\right.
$$

After this do a matrix again:

$$
A=\left[\begin{array}{lll}
x_{4}-x_{1} & y_{4}-y_{1} & z_{4}-z_{1} \\
x_{4}-x_{2} & y_{4}-y_{2} & z_{4}-z_{2} \\
x_{4}-x_{3} & y_{4}-y_{3} & z_{4}-z_{3}
\end{array}\right], X=\left[\begin{array}{l}
x \\
y \\
z
\end{array}\right], b=\left[\begin{array}{l}
\frac{1}{2}\left[d_{1}^{2}-d_{4}^{2}+x_{4}^{2}+y_{4}^{2}+z_{4}^{2}-\left(x_{1}^{2}+y_{1}^{2}+z_{1}^{2}\right)\right] \\
\frac{1}{2}\left[d_{2}^{2}-d_{4}^{2}+x_{4}^{2}+y_{4}^{2}+z_{4}^{2}-\left(x_{2}^{2}+y_{2}^{2}+z_{2}^{2}\right)\right] \\
\frac{1}{2}\left[d_{3}^{2}-d_{4}^{2}+x_{4}^{2}+y_{4}^{2}+z_{4}^{2}-\left(x_{3}^{2}+y_{3}^{2}+z_{3}^{2}\right)\right]
\end{array}\right]
$$

The problem is simplified to:

$$
A X=b
$$
following formula:

$$
\left(A^{T} A^{-1}\right) A^{T} X=b
$$


Therefore according to the last equation and the actual situation, the optimization coordinate of the transmitter ${ }^{T(x, y, z)}$ can be calculated.

\subsection{Universal Wheels Control Based on PI Controller}

According to other paper [11], the relationship between the rotating speed $\left(v_{1}, v_{2}, v_{3}\right)$ and the velocity component on the axis $\left(v_{x}, v_{y}, v_{z}\right)$ together with the angular velocity of the carrier $(\omega)$ is like this:

$$
\left(\begin{array}{l}
v_{1} \\
v_{2} \\
v_{3}
\end{array}\right)=\left(\begin{array}{ccc}
\sin (\phi / 2) & \cos (\phi / 2) & L \\
-\sin (\phi / 2) & \cos (\phi / 2) & L \\
0 & -1 & L
\end{array}\right)\left(\begin{array}{l}
v_{x} \\
v_{y} \\
\omega
\end{array}\right)
$$

After getting the speed of the wheels, the maintaining of the velocity of each wheel is the next problem. Since there is friction on the ground and friction at different places is also variant, the desired velocity must be maintained. Therefore, PID algorithm is adopted.

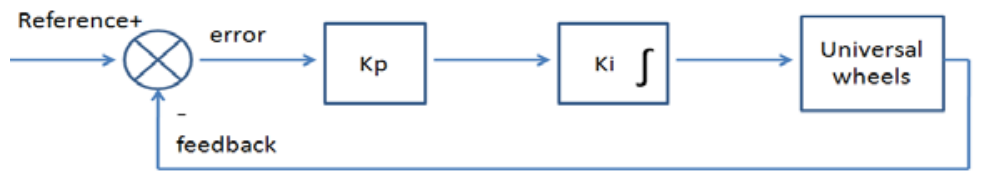

Fig.3 Structure of PI controller

As the figure 3, with the received reference, calculate the error between output and reference. Then put it into the PI controller and pwm can be maintained at a certain value approximately. It can be realized using the formula:

$$
p w m=e(k) \times K_{p}+K_{i} \sum e(k)
$$

\section{Experimentation and Results}

\subsection{Experiments and Results on PI}

In order to test whether the PI algorithm works as expected, this paper has designs an experiment which is shown in the graphs on how the actual velocity (blue) acts when the desired velocity (red) changes dramatically. The results are shown in figure 4 and figure 5:

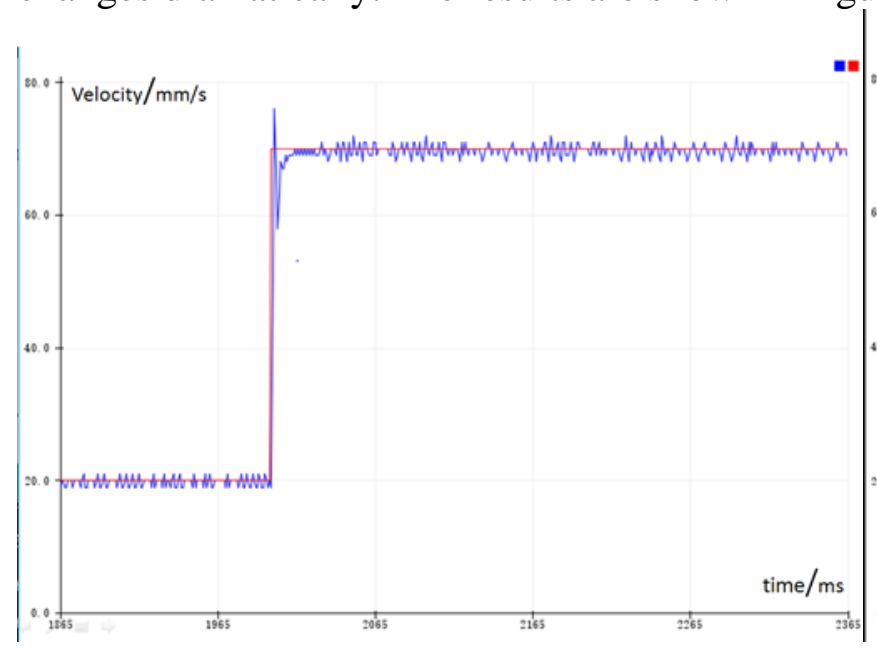

Fig. 4 Dynamic response of step up

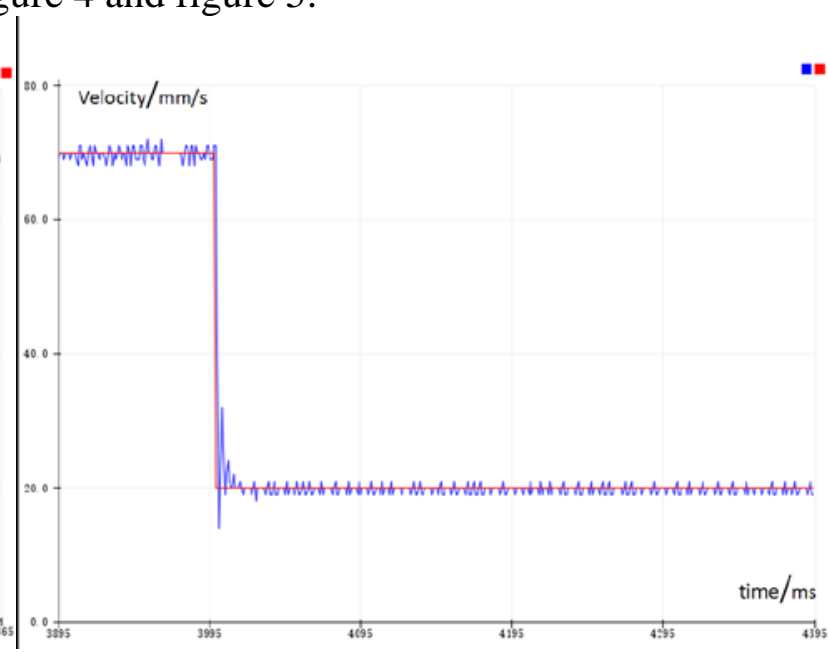

Fig. 5 Dynamic response of step down

From the graphs, the steady state is stable; the output tracking effect is satisfying, while in the dynamic state the actual velocity responds very fast. Therefore the PI controller is effective.

\subsection{Experiments and Results on Ultrasonic Positioning}

For testing the efficiency of the ultrasonic positioning system, this paper has come up with an experiment (figure 6) by putting a LCD screen which shows the distance between each ultrasonic receivers and the ultrasonic transmitter, on the top of the carrier. 
Then, according to the values showed on the screen (figure 7) and the equation derived in section three, a group of data can be deducted out as table 1

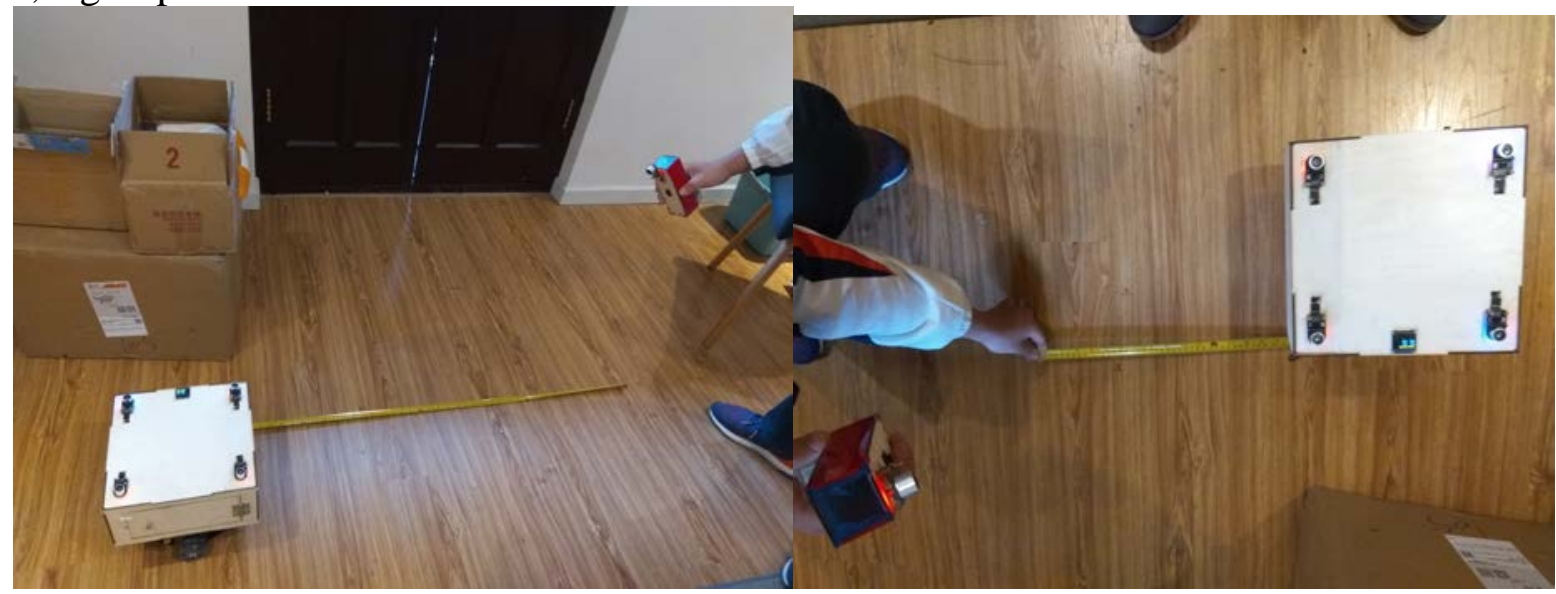

Fig.6 The experiments set up

Fig.7 The experiments result shows in LCD

From the data in the Table 1, a certain result is shown by using every three receivers. While the four receivers can be listed in four results, by using the least squares optimization method, a minimal value of the coordinate is worked out. Therefore the ultrasonic positioning algorithm is proved accurately enough.

Table 1 Analysis of experimental results

\begin{tabular}{|c|c|c|c|}
\hline Actual coordinates & Number of tests & $\begin{array}{c}\text { Average } \\
\text { coordinates result } \\
\text { of calculation }\end{array}$ & $\begin{array}{c}\text { Average error of } \\
\text { distance }\end{array}$ \\
\hline$(100,0)$ & 100 & $(98.3,2.2)$ & $2.2 \%$ \\
\hline$(0,100)$ & 100 & $(-1.9,99.3)$, & $0.7 \%$ \\
\hline
\end{tabular}

\section{Conclusions}

In conclusion, this paper illustrates how UPASAFLEC (Ultrasonic Positioning and Analysis System for Automatic following and Locating Electric Carrier) works. It uses an ultrasonic positioning algorithm and PI velocity controller in detail. The experiments results show the UPASAFLEC successful. The accuracy of the positioning system and the clever use of the PI controller make the project relatively unique and better than other projects on this area up to now .

\section{Acknowledgments}

This work was supported by Mengfu Education Technology Co.Ltd.

\section{References}

[1] Fukuju Y, Minami M, Morikawa H, et al. DOLPHIN: An Autonomous Indoor Positioning System in Ubiquitous Computing Environment[J]. WSTFES, 2003, 3: 53.

[2]Khyam M O, Ge S S, Li X, et al. Pseudo-Orthogonal Chirp-Based Multiple Ultrasonic Transducer positioning [J]. IEEE Sensors Journal, 2017, 17(12): 3832-3843.

[3] McCarthy M R, Muller H L. RF free ultrasonic positioning[C]//null. IEEE, 2003: 79.

[4]Khyam M O, Ge S, Xinde L, et al. Mobile Ultrasonic Transducer Positioning[J]. The Journal of Engineering, 2017, 1(1).

[5]Jian Xong, Xianzan Zhou, Hang Guo,Weisheng Zhong. An Improved Active Bat Indoor Ultrasonic Positioning Method [J].Journal of Detection \& Control , 2017, 39(1):101-105. 
[6] Lei Cai, Tingting Zhou, Yunpeng Guo, et al. Intelligent Following Carriage Based on Ultrasonic positioning [J]. Electronic Measurement Technology, 2013, 36(11):76-79.

[7] Yuhang Tian, Xudong Zhang, Zhiyuan Chen, et al. Blind Guiding System Based on Ultrasonic Positioning [J].Electronic Technology \& Software Engineering, 2017(7):114-115.

[8]MENG Fan-kun, JU Yong-feng, WEN Li-min, WEN Chang-bao, et al. Moving Target Tracking Algorithm Based on APRBA Particle Filter Algorithm [J]. Control Engineering of China, 2017, 24(6):1212-1217.

[9] Li Xinlei, Qin Jianjun, Wu Boyuo, et al. Control of zero turning tadius independent following robot based on ultrasonic positioning $[\mathrm{J}]$. Science Technology and Engineering, 2017, 17( 14) : 228-233

[10]Li Bing. Design and Implementation of Indoor Location and Navigation Device Based on Ultrasonic [D].Harbin University of Science and Technology, 2017.

[11]Zhichen Xie. Three-wheel Omni-directional Mobile Robot Motion Controls [D]. Institutes of Technology of Changsha, 2010. 